\title{
ON CLASSICAL CLIFFORD THEORY
}

\author{
MORTON E. HARRIS
}

Dedicated to Professor B. Huppert on the occasion of his sixtieth birthday, October 22, 1987

\begin{abstract}
Let $k$ be a field, let $N$ be a normal subgroup of a finite group $H$ and let $M$ be a completely reducible $k[N]$-module. We give sufficient conditions for a finite dimensional (finite) group crossed product $k$-algebra to be a Frobenius or symmetric $k$-algebra. These results imply that $k[H] /(J(k[N]) k[H])$ and the endomorphism $k$-algebra, $\operatorname{End}_{k[H]}\left(M^{H}\right)$, of the induced module $M^{H}$ are symmetric $k$-algebras. We also completely describe the $k[H]$-indecomposable decomposition of $M^{H}$. It follows that the head and socle of an indecomposable component of $M^{H}$ are irreducible isomorphic $k[H]$-modules.
\end{abstract}

1. Introduction and statements. Our notation and terminology are standard and tend to follow the conventions of $[\mathbf{4}, \mathbf{6}$ and 8]. In particular, in this article, all rings have identities, all modules over a ring are right and unital, all vector spaces and algebras have finite dimension over the stipulated field and if $n$ is a positive integer and $V$ is a module, then $n V$ denotes the module direct sum of $n$ copies of $V$.

Throughout this article $G$ denotes a finite group, $R$ denotes a nonzero ring and $U(R)$ denotes the multiplicative group of units of $R$.

The ring $R$ is $G$-graded if $R$ is a direct sum $R=\bigoplus_{g \in G} R_{g}$ of additive subgroups $R_{g}$, one for each $g \in G$, such that $R_{g} R_{h} \leq R_{g h}$ for all $g, h \in G$. In that case, the subgroup $R_{1}$ corresponding to the identity $1_{G}$ of $G$ is a subring and contains the identity 1 of $R$ (cf. [4, Proposition 1.4]) and $R_{g}$ is an $\left(R_{1}, R_{1}\right)$-bimodule for all $g \in G$. Also if $R_{g} R_{h}=R_{g h}$ for all $g, h \in G$, then $R$ is said to be fully $G$-graded (this terminology conforms to [5, $\S 1]$ and differs from [4]). If $R$ is also an algebra over the commutative ring $\mathscr{O}$ and if $R_{g}$ is an $\mathscr{O}$-submodule for all $g \in G$, then $R$ is called a $G$-graded $\mathscr{O}$-algebra.

For the $G$-graded ring $R \neq(0)$, if $g \in G$ and $0 \neq x \in R_{g}$, then we call $g$ the degree of $x$ and write $\operatorname{deg}(x)=g$. A unit $u \in U(R)$ is said to be graded if $u \in R_{g}$ for some $g \in G$; in which case $u^{-1} \in R_{g-1}$ by [4, Lemma 5.1]. The set $\operatorname{Gr} U(R)=\bigcup_{g \in G}\left(U(R) \cap R_{g}\right)$ of graded units of $R$ is a subgroup of $U(R)$ and clearly $\operatorname{deg}: \operatorname{Gr} U(R) \rightarrow G$ is a group homomorphism with $\operatorname{Ker}(\operatorname{deg})=U\left(R_{1}\right)$. Thus we have a sequence of group homomorphisms:

$$
1 \rightarrow U\left(R_{1}\right) \stackrel{i}{\rightarrow} \operatorname{Gr} U(R) \stackrel{\operatorname{deg}}{\rightarrow} G \rightarrow 1
$$

where $i$ denotes the canonic inclusion map and where the sequence is exact except possibly at $G$. Also conjugation in $R$ defines a group action of $\operatorname{Gr} U(R)$ on $R_{1}: r_{1}^{u}=u^{-1} r_{1} u$ for all $r_{1} \in R_{1}$ and $u \in \operatorname{Gr} U(R)$, so that conjugation induces a

Received by the editors August 28, 1987.

1980 Mathematics Subject Classification (1985 Revision). Primary 20C20.

(C) 1988 American Mathematical Society $0002-9947 / 88 \$ 1.00+\$ .25$ per page 
homomorphism of the group $\operatorname{Gr} U(R)$ into the automorphism group $\operatorname{Aut}\left(R_{1}\right)$ of the subring $R_{1}$ and a homomorphism of $\operatorname{Gr} U(R)$ into $\operatorname{Aut}\left(Z\left(R_{1}\right)\right)$.

By definition, the $G$-graded ring $R=\bigoplus_{g \in G} R_{g}$ is called a $G$-crossed product if the sequence (1.1) is exact (or equivalently: if $U(R) \cap R_{g} \neq \varnothing$ for all $g \in G$ ).

Assume that $R$ is a $G$-crossed product, choose $\beta_{g} \in U(R) \cap R_{g}$ for each $g \in G$ where $\beta_{1}=1_{R}$ and let $\pi: \operatorname{Gr} U(R) \rightarrow \operatorname{Aut}\left(Z\left(R_{1}\right)\right)$ denote the group homomorphism induced by the conjugation action of $\operatorname{Gr} U(R)$ on $Z\left(R_{1}\right)$. Here we have: $R_{g}=R_{1} \beta_{g}=\beta_{g} R_{1}, U(R) \cap R_{g}=U\left(R_{1}\right) \beta_{g}=\beta_{g} U\left(R_{1}\right)$ and if $r \in Z\left(R_{1}\right)$ and $u \in U\left(R_{1}\right)$, then $r^{\pi\left(u \beta_{g}\right)}=r^{u \beta_{g}}=r^{\beta_{g}}$ for all $g \in G$. Thus $U\left(R_{1}\right) \leq \operatorname{Ker}(\pi)$ and the exact sequence (1.1) yields a group action of $G$ on $Z\left(R_{1}\right)$.

We now proceed directly to state our first two main results.

As above, let $R$ be a $G$-crossed product and assume that $E$ is a $G$-invariant subfield of $Z\left(R_{1}\right)$ such that $\operatorname{dim}_{E}\left(R_{1}\right)$ is finite so that $R_{1}$ is a finite dimensional $E$-algebra. Let $F=E^{G}$ denote the $G$-fixed subfield of $E$ and let $\pi^{*}: G \rightarrow \operatorname{Aut}(E)$ denote the group homomorphism induced by $\pi$ and restriction to $E$. We conclude that $E / F$ is a finite Galois extension and $\operatorname{Gal}(E / F)=\pi^{*}(G)$ by a Theorem of Artin (cf. [9, VIII, Theorem 1.8]). Clearly $F=Z(R) \cap E \leq Z(R) \cap R_{1}$.

Let $K$ be a subfield of $F=E^{G}$ such that $F / K$ is a finite field extension and let $T=\operatorname{Tr}_{F}^{E}: E \rightarrow F$ denote the $F$-linear trace map. Also let $0 \neq \lambda \in \operatorname{Hom}_{K}(F, K)$. Since $E / F$ is a finite separable field extension, we have $T(E)=F$ by [9, VIII, Theorem 5.2] and hence $\lambda(T(E))=K$. Moreover $R$ is a $G$-crossed product finite dimensional $K$-algebra since $K \leq F=Z(R) \cap E \leq Z(R) \cap R_{1}$.

Fix $\varphi \in \operatorname{Hom}_{E}\left(R_{1}, E\right)$ and define $f: R \rightarrow K$ by

if $x=\sum_{g \in G} x_{g} \in R$ for unique elements $x_{g} \in R_{g}$ for all $g \in G$, set $f(x)=$ $\lambda\left(T\left(\varphi\left(x_{1}\right)\right)\right)$.

Clearly $f \in \operatorname{Hom}_{K}(R, K)$.

LEMMA 1. Assume that $\operatorname{Ker}(\varphi)$ contains no nonzero right ideal of $R_{1}$ (so that $R_{1}$ is a Frobenius E-algebra by [8, VII, Exercise 53]). Then

(a) $\operatorname{Ker}(f)$ contains no nonzero right ideal of $R$ and $R$ is a Frobenius $K$-algebra; and

(b) if $\varphi\left(x_{1} y_{1}\right)=\varphi\left(y_{1} x_{1}\right)$ for all $x_{1}, y_{1} \in R_{1}$ and if $\varphi\left(x_{1}^{u}\right)=\varphi\left(x_{1}\right)^{\pi(u)}$ for all $x_{1} \in R_{1}$ and all $u \in \operatorname{Gr} U(R)$, then $f(x y)=f(y x)$ for all $x, y \in R$ and $R$ is a symmetric $K$-algebra.

Note that Lemma 1(a) is already known for it is a special case of $[\mathbf{1 0}$, Satz 6]. We shall utilize Lemma 1 (b) to prove

Proposition 2. Let $K$ be a field and let $R=\bigoplus_{g \in G} R_{g}$ be a finite dimensional $G$-crossed product $K$-algebra such that $R_{1}$ is a semisimple $K$-algebra. Then $R$ is a symmetric $K$-algebra.

This proposition generalizes a well known result of Eilenberg and Nakayama (cf. [2, Proposition 9.8]). Our proof of this proposition uses the reduced trace (cf. [2, $\S 7 \mathrm{D}])$.

Again let $K$ denote an arbitrary field.

Next we present an example due to E. C. Dade of a finite dimensional symmetric group-graded crossed product $K$-algebra with a 1-component that is not a symmetric $K$-algebra. 
EXAmple 3 (E. C. DADE). Let $K[X]$ denote the polynomial ring over $K$ in 1 independent variable $X$ and let $K[x]=K[X] /\left(X^{2}\right)$ denote the truncated polynomial $K$-algebra where $x=X+\left(X^{2}\right)$. Also let $R$ denote the $K$-algebra of all $2 \times 2$ matrices over $k[x]$ and let $e_{i j}$ for $1 \leq i, j \leq 2$ denote the usual matrix "units" of $R$. Thus $x e_{i j}=e_{i j} x$ for all $1 \leq i, j \leq 2, x^{2}=0$ and $R$ has $K$-basis $\left\{e_{i j}, x e_{i j} \mid 1 \leq\right.$ $i, j \leq 2\}$. Let $G=\langle g\rangle$ be a cyclic group of order 2 and set $R_{1}=K e_{11}+K e_{22}+$ $K x e_{12}+K x e_{21}$ and $R_{g}=K e_{12}+K e_{21}+K x e_{11}+K x e_{22}$. It is straightforward to verify that $R$ is then a $G$-graded finite dimensional $K$-algebra. Also $\left(e_{21}+e_{12}\right) \in R_{g}$ and $\left(e_{21}+e_{12}\right)^{2}=e_{11}+e_{22}=1_{R}$, so that $R$ is a $G$-crossed product. Moreover $R$ is a symmetric $K$-algebra by [8, VII, Exercises 48 and 51]. It is easy to see that $J\left(R_{1}\right)=K x e_{21}+K x e_{12}$ and that $R_{1}=\left(e_{11} R_{1}\right) \oplus\left(e_{22} R_{1}\right)$ in $\operatorname{Mod}\left(R_{1}\right)$. Set $P_{1}=e_{11} R_{1}$. Thus $P_{1}=e_{11} R_{1}=K e_{11}+K x e_{12}$ is a projective $R_{1}$-module and $P_{1} J\left(R_{1}\right)=K x e_{12}=\operatorname{Rad}\left(P_{1}\right)$. Here $\operatorname{dim}_{K}\left(P_{1} / \operatorname{Rad}\left(P_{1}\right)\right)=\operatorname{dim}_{K}\left(\operatorname{Rad}\left(P_{1}\right)\right)=1$, $P_{1}$ is indecomposable, $e_{11} \in \operatorname{Ann}_{R_{1}}\left(\operatorname{Rad}\left(P_{1}\right)\right)$ and $e_{11} \notin \operatorname{Ann}_{R_{1}}\left(P_{1} / \operatorname{Rad}\left(P_{1}\right)\right)$. Thus $P_{1} / \operatorname{Rad}\left(P_{1}\right)$ and $\operatorname{Rad}\left(P_{1}\right)$ are not isomorphic in $\operatorname{Mod}\left(R_{1}\right)$ and hence $R_{1}$ is not a symmetric $K$-algebra by [8, VII, Theorem 11.6(c)].

For the remainder of this section, let $k$ denote an arbitrary field, let $H$ denote an arbitrary finite group and let $N$ be an arbitrary normal subgroup of $H$. Here $k[N]$ and $k[H]$ denote the associated group algebras, $\operatorname{Mod}(k[N])$ and $\operatorname{Mod}(k[H])$ are the abelian categories of finitely generated $k[N]$ and $k[H]$-modules, respectively, and $J(k[N])$ and $J(k[H])$ denote the Jacobson radicals of $k[N]$ and $k[H]$, respectively.

As is well known, (cf. [8, VII, Theorem 7.21]), $J(k[N]) k[H]=k[H] J(k[N])$, $J(k[N]) k[H]$ is an ideal of $k[H]$ and $J(k[N]) k[H] \leq J(k[H])$.

Let $V$ be a $k[H]$-module and let $S$ be a subset of $k[H]$. Then

$$
V J(k[N])=V J(k[N]) k[H]
$$

$V J(k[N])$ is a $k[H]$-submodule of $V$ and $V J(k[N]) \leq V J(k[H])$. Also $\operatorname{Ann}_{V}(S)=$ $\{v \in V \mid v S=(0)\}$ and $\operatorname{Ann}_{V}(S)$ is a $k[H]$-submodule of $V$ if $S$ is a left ideal of $k[H]$. Moreover $\mathscr{H}(V)=V /(V J(k[H]))$ denotes the head of $V$ and $\mathscr{S}(V)=$ $\operatorname{Ann}_{V}(J(k[H]))$ denotes the socle of $V$. Clearly $\mathscr{H}(V) \cong \mathscr{H}(V /(V J(k[N])))$ in $\operatorname{Mod}(k[H]), \operatorname{Ann}_{V}(J(k[N]))=\operatorname{Ann}_{V}(J(k[N]) k[H])$ and

$$
\mathscr{S}(V)=\mathscr{S}\left(\operatorname{Ann}_{V}(J(k[N]))\right) .
$$

Let $\operatorname{Irr}(k[H])$ denote a complete system of representatives of the isomorphism classes of irreducible $k[H]$-modules and, for each $L \in \operatorname{Irr}(k[H])$, let $P(L)$ denote a projective cover of $L$. Here, for $L \in \operatorname{Irr}(k[H])$, we have

$$
\mathscr{H}(P(L)) \cong \mathscr{H}(P(L) /(P(L) J(k[N]))) \cong \mathscr{S}(P(L))=\mathscr{S}\left(\operatorname{Ann}_{P(L)}(J(k[N]))\right)
$$

in $\operatorname{Mod}(k[H])$ by [8, VII, Theorems 11.2 and 11.6(c)]. Thus $P(L) /(P(L) J(k[N]))$ and $\operatorname{Ann}_{P[L]}(J(k[N]))$ are indecomposable $k[H]$-modules. Also $\{P(L) \mid L \in$ $\operatorname{Irr}(k[H])\}$ is a complete set of representatives for the isomorphism classes of projective indecomposable $k[H]$-modules, cf. [8, VII, Theorem 10.9].

For any $k[H]$-module $V$ and any $L \in \operatorname{Irr}(k[H])$, let $\operatorname{mult}(L$ in $V)$ denote the multiplicity of $L$ as a composition factor of $V$.

Next we present our main results in classical Clifford Theory of Finite Group Representation Theory. 
THEOREM 4. $k[H] /(J(k[N]) k[H])$ is a symmetric $k$-algebra.

THEOREM 5. Let $W$ be a completely reducible $k[N]$-module. Then $\operatorname{End}_{k[H]}\left(W^{H}\right)$ is a finite dimensional symmetric $k$-algebra.

Note that the $N=1$ case of Theorem 4 is the well-known fact that $k[H]$ is a symmetric algebra (cf. [8, VII, Theorem 11.2]). Also, as in Theorem 5, $\operatorname{End}_{k[H]}\left(W^{H}\right)$ plays a basic role in classical stable Clifford theory (cf. [4, §8]).

Theorem 4 also has implications for $k[N]$-projective $k[H]$-modules:

Proposition 6. Let $W$ be a $k[N]$-projective $k[H]$-module and let $r$ be a positive integer such that $W J(k[N])^{r}=(0)$. Then $W=W J(k[N])^{0} \geq W J(k[N])^{1} \geq$ $\cdots \geq W J(k[N])^{r-1} \geq W J(k[N])^{r}=(0)$ is a $k[H]$-filtration of $W$ to (0) where the filtration factors $\left(W J(k[N])^{j}\right) /\left(W J(k[N])^{j+1}\right)$ are projective modules over the symmetric $k$-algebra $k[H] /(J(k[N]) k[H])$ for all $0 \leq j \leq r-1$.

Let $W$ be a completely reducible $k[N]$-module and consider the direct sum decomposition of $W^{H}$ into indecomposable $k[H]$-modules. Since induction is an additive functor, it suffices to study this problem for a fixed (but arbitrary) irreducible $k[N]$-module $M$.

Let $P(M)$ be a projective cover of $M$ in $\operatorname{Mod}(k[N])$ and let $\operatorname{Irr}(k[H] \mid M)=\{L \in$ $\operatorname{Irr}(k[H]) \mid M$ is isomorphic to a composition factor (and hence to a summand) of $\left.L_{N}\right\}$. As is well known, (cf. [8, VII, Theorem 4.13(a)]), for any $L \in \operatorname{Irr}(k[H])$, we have

$$
\begin{aligned}
& \operatorname{mult}\left(L \text { in } \mathscr{H}\left(M^{H}\right)\right) \operatorname{dim}_{k}\left(\operatorname{End}_{k[H]}(L)\right) \\
& \quad=\operatorname{mult}\left(M \text { in } L_{N}\right) \operatorname{dim}_{k}\left(\operatorname{End}_{k[N]}(M)\right) .
\end{aligned}
$$

Theorem 4 is used in our proof of part (c) (ii) of our next main result which describes the complete indecomposable decomposition of $M^{H}$ and $P(M)^{H}$ in $\operatorname{Mod}(k[H])$ :

THEOREM 7. (a)

$$
P(M)^{H} \cong \bigoplus_{L \in \operatorname{Irr}(k[H] \mid M)}\left(\left(\operatorname{mult}\left(L \text { in } \mathscr{H}\left(M^{H}\right)\right)\right) P(L)\right)
$$

(b)

$$
M^{H} \cong \bigoplus_{L \in \operatorname{Irr}(k[H] \mid M)}\left(\left(\operatorname{mult}\left(L \text { in } \mathscr{H}\left(M^{H}\right)\right)\right)(P(L) /(P(L) J(k[N])))\right)
$$

and

(c) if $L \in \operatorname{Irr}(k[H] \mid M)$, then

(i) $P(L) /(P(L) J(k[N])) \cong \operatorname{Ann}_{P(L)}(J(k[N]))$,

(ii) $\mathscr{H}(P(L) /(P(L) J(k[N]))) \cong L \cong \mathscr{S}(P(L) /(P(L) J(k[N])))$ and

(iii) $P(L) /(P(L) J(k[N]))$ is indecomposable, in $\operatorname{Mod}(k[H])$.

Next we present three applications of Theorem 7 .

COROLLARY 8. Let $L \in \operatorname{Irr}(k[H])$. The following two conditions are equivalent:

(a) $L$ is $k[N]$-projective; and

(b) $P(L) J(k[N])=P(L) J(k[H])$.

Our second application gives a combination with alternate proofs of $[8$, Theorems 7.21(b) and (c) and 9.4]: 
COROLLARY 9. The following three conditions are equivalent:

(a) $J(k[N]) k[H]=J(k[H])$;

(b) if $W$ is a completely reducible $k[N]$-module, then $W^{H}$ is a completely reducible $k[H]$-module;

(c) $\operatorname{char}(k)$ does not divide $|H / N|$.

In that case, if $M$ is an irreducible $k[N]$-module and $L$ is an irreducible $k[H]$ module, then $L \mid M^{H}$ if and only if $M \mid L_{N}$.

Let $A(N)=\sum_{n \in N} k(n-1)$ denote the augmentation ideal of $k[N]$, so that $A(N)=\operatorname{Ann}_{k[N]}\left(1_{N}\right)$. Part (b) of our final result is related to [8, VII, Exercise 18(b)]:

COROLLARY 10. Let $L$ be an irreducible $k[H]$-module with $N \geq \operatorname{Ker}(L)$. View $L$ as an irreducible $k[H / N]$-module, let $Q(L)$ denote a projective cover of $L$ in $\operatorname{Mod}(k[H / N])$ and view $Q(L)$ as a $k[H]$-module with $N \leq \operatorname{Ker}(Q(L))$. Then

(a) $Q(L) \cong P(L) /(P(L) J(k[N]))$ in $\operatorname{Mod}(k[H])$; and

(b) $P(L) J(k[N])=P(L) A(N)$.

In $\S 2$, we present some preliminary results. These results are used in $\S 3$ to prove all of our main results.

Finally, the author would like to thank Professor Everett C. Dade of the University of Illinois-Urbana, Professor Reinhard Knörr of the University of Essen and Dr. Burkhard Külshammer of the University of Dortmund for stimulating discussions about this paper.

2. Preliminary results. For our first result in this section, let $R$ be a ring, let $J$ be a subset of $R$, let $I$ be a right ideal of $R$, let $X$ be an $R$-module and let $X=\bigoplus_{s \in S} W_{s}$ be a direct sum $R$-module decomposition of $X$. We trivially have

LEMMA 2.1. (a) $X I$ and $W_{s} I$ for all $s \in S$ are $R$-submodules of $X$ and $X I=$ $\bigoplus_{s \in S}\left(W_{s} I\right)$

(b) $X /(\because I) \cong \bigoplus_{s \in S}\left(W_{s} / W_{s} I\right)$ in $\operatorname{Mod}(R)$; and

(c) $\operatorname{Ann}_{X}(J)=\bigoplus_{s \in S} \operatorname{Ann}_{W_{s}}(J)$.

LEMMA 2.2. Let $K$ be a field, let $R$ be a finite dimensional symmetric $K$ algebra and let $V$ be a finitely generated projected $R$-module. If $V$ is not a completely reducible $R$-module, then $V>\operatorname{Rad}(V) \geq \operatorname{Rad}(V) \cap \operatorname{Soc}(V)>(0)$ is an $R$-filtration of $V$ and some $R$-composition factor of $V$ occurs in both $V / \operatorname{Rad}(V)$ and $\operatorname{Rad}(V) \cap$ $\operatorname{Soc}(V)$.

PROOF. Clearly it suffices to assume that $V$ is a projective indecomposable $R$-module and then the desired conclusion follows from [8, VII, Theorem 11.6].

For the next result, let $A, B$ be rings and let $\sigma: A \rightarrow B$ be a ring isomorphism. Let $K$ be a subfield of $Z(A)$ such that $A$ is a finite dimensional separable $K$-algebra. Set $L=K^{\sigma}$. Thus $L$ is a subfield of $Z(B), B$ is a finite dimensional separable $L$ algebra and $\operatorname{dim}_{K}(A)=\operatorname{dim}_{L}(B)$.

We shall, for the time being, adhere to the notation of $[2, \S 7 \mathrm{D}]$.

Let $d \in A$ and let red. char. poly ${ }_{A / K}(d)=X^{n}+k_{1} X^{n-1}+\cdots+k_{n-1} X+k_{n}$ where $n$ is a positive integer and $k_{i} \in K$ for all $1 \leq i \leq n$. 
LEMMA 2.3. red. char. poly ${ }_{B / L}\left(d^{\sigma}\right)=X^{n}+\left(k_{1}^{\sigma}\right) X^{n-1}+\cdots+\left(k_{n-1}^{\sigma}\right) X+k_{n}^{\sigma}$.

Proof. Let $\bar{K}, \bar{L}$ denote algebraic closures of $K$ and $L$, respectively. As is well known, (cf. [9, VII, Theorem 2.8]), $\sigma$ can be extended to a field isomorphism $\bar{\sigma}: \bar{K} \rightarrow \bar{L}$ and hence there is a ring isomorphism $\tau: \bar{K} \otimes_{K} A \rightarrow \bar{L} \otimes_{L} B$ such that $\tau\left(\bar{k} \otimes_{K} a\right)=\left(\bar{k}^{\bar{\sigma}}\right) \otimes_{L}\left(a^{\sigma}\right)$ for all $\bar{k} \in \bar{K}$ and all $a \in A$. Since $\bar{K} \otimes_{K} A$ is a finite dimensional semisimple $\bar{K}$-algebra, there is a $\bar{K}$-algebra isomorphism $h: \bar{K} \otimes_{K} A \rightarrow \bigoplus_{i=1}^{m} M_{r_{i}}(\bar{K})$ for some positive integer $m$ and some positive integers $r_{i}$ for all $1 \leq i \leq m$. Let $\rho: \bigoplus_{i=1}^{m} M_{r_{i}}(\bar{K}) \rightarrow \bigoplus_{r=1}^{m} M_{r_{i}}(\bar{L})$ denote the ring isomorphism induced by $\bar{\sigma}: \bar{K} \rightarrow \bar{L}$. Set $\gamma=\rho \circ h \circ \tau^{-1}: \bar{L} \otimes_{L} B \rightarrow \bigoplus_{i=1}^{m} M_{r_{i}}(\bar{L})$, so that $\gamma$ is an $\bar{L}$-algebra isomorphism and $\gamma \circ \tau=\rho \circ h$. Let $h\left(1 \otimes_{K} d\right)=\bigoplus_{i=1}^{m} \varphi_{i}(d)$ for unique matrices $\varphi_{i}(d) \in M_{r_{i}}(\bar{K})$ for all $1 \leq i \leq m$, so that

$$
\text { red. char. poly }{ }_{A / K}(d)=\prod_{i=1}^{m} \operatorname{char} \text {. poly. }\left(\varphi_{i}(d)\right) \text {. }
$$

Also let $\gamma\left(1 \otimes_{L} d^{\sigma}\right)=\bigoplus_{i=1}^{m} \psi_{i}\left(d^{\sigma}\right)$ for unique matrices $\psi_{i}\left(d^{\sigma}\right) \in M_{r_{i}}(\bar{L})$ for all $1 \leq i \leq m$, so that

$$
\text { red. char. poly }{ }_{B / L}\left(d^{\sigma}\right)=\prod_{i=1}^{m} \text { char. poly. } \psi_{i}\left(d^{\sigma}\right) .
$$

Here

$$
\begin{aligned}
\rho\left(h\left(1 \otimes_{K} d\right)\right) & =\bigoplus_{i=1}^{m}\left(\varphi_{i}(d)^{\rho}\right)=\gamma(\tau(1 \otimes d)) \\
& =\gamma\left(1 \otimes d^{\sigma}\right)=\prod_{i=1}^{m} \psi_{i}\left(d^{\sigma}\right) .
\end{aligned}
$$

Hence $\varphi_{i}(d)^{\rho}=\psi_{i}\left(d^{\sigma}\right)$ for all $1 \leq i \leq m$, (char. poly. $\left.\left(\varphi_{i}(d)\right)\right)^{\bar{\sigma}}=$ char. poly. $\left(\psi_{i}\left(d^{\sigma}\right)\right)$ for all $1 \leq i \leq m$ and the desired conclusion follows.

Our next result is presented without its straightforward proof.

LEMMA 2.4. Let $\mathscr{O}$ be a commutative ring and let $R=\bigoplus_{g \in G} R_{g}$ be a $G$ crossed product $\mathscr{O}$-algebra. Also let $\left\{e_{i} \mid 1 \leq i \leq n\right\}$ be a set of $G$-fixed orthogonal idempotents of $Z\left(R_{1}\right)$ such that $1=\sum_{i=1}^{n} e_{i}$. (Clearly $e_{i} \in Z(R) \cap R_{1}$ for all $1 \leq i \leq n)$. Choose $\beta_{g} \in U(R) \cap R_{g}$ for all $g \in G$. Then $R=\bigoplus_{i=1}^{n}\left(e_{i} R\right)$ is a direct sum decomposition of $R$ into ideals $e_{i} R$ where each $e_{i} R$ is a $G$-crossed product $\mathscr{O}$-algebra such that for all $1 \leq i \leq n$ :

(a) $e_{i}$ is the identity of $e_{i} R$;

(b) $\left(e_{i} R\right)_{g}=e_{i} R_{g}$ for all $g \in G$;

(c) $e_{i} \beta_{g} \in U\left(e_{i} R\right) \cap\left(\left(e_{i} R\right)_{g}\right)$ for all $g \in G$; and

(d) $U(R)=\bigoplus_{i=1}^{n} U\left(e_{i} R\right)$.

LEMMA 2.5. Let $R$ be a fully $G$-graded ring such that $R_{1}$ is a semisimple ring (in the sense of $[\mathbf{1}, \mathrm{I}, \S 4]$ ). Then every $G$-graded $R$-module is projective.

Proof. Let $M$ be a $G$-graded $R$-module. Then $M \cong M_{1} \otimes_{R_{1}} R$ in $\operatorname{Gr} \operatorname{Mod}(R)$ by [4, Theorem 2.8]. Here $M_{1}$ is a projective $R_{1}$-module and [1, II, Proposition 6.1] implies that $M$ is a projective $R$-module. Q.E.D. 
COROLlARY 2.6. Let $R$ be a fully $G$-graded ring such that $R_{1} / J\left(R_{1}\right)$ is a semisimple ring (in the sense of $[1, \mathrm{I}, \S 4])$. Set $I=J\left(R_{1}\right) R$, so that $I$ is a (twosided) $G$-graded ideal by [3, Proposition 1.11] and $I \leq J(R)$ by $[\mathbf{7}$, Lemma 2.7(b)]. Let $M$ be a $G$-graded $R$-module and let $N \mid M$ in $\operatorname{Mod}(R)$. Then, for each integer $j \geq 0,\left(N I^{j}\right) /\left(N I^{j+1}\right)$ is a projective $R / I$-module.

PROOF. Fix an integer $j \geq 0$. Clearly $M I^{j}$ is a $G$-graded $R$-module with $\left(M I^{j}\right)_{g}=M_{g} J\left(R_{1}\right)^{j}$ for all $g \in G$ and $N I^{j} \mid M I^{j}$ in $\operatorname{Mod}(R)$. Thus

$$
\left(\left(N I^{j}\right) /\left(N I^{j+1}\right)\right) \mid\left(\left(M I^{j}\right) /\left(M I^{j+1}\right)\right)
$$

in $\operatorname{Mod}(R / I)$. However $R / I$ is a fully $G$-graded $\operatorname{ring}$ with $(R / I)_{1} \cong R_{1} / J\left(R_{1}\right)$ as rings and $\left(M I^{j}\right) /\left(M I^{j+1}\right)$ is a $G$-graded $R / I$-module. Thus Lemma 2.5 and the fact that summands of projective modules are projective yield the desired conclusion.

For the remainder of this section, let $k$ denote an arbitrary field, let $H$ denote an arbitrary finite group and let $N$ denote an arbitrary normal subgroup of $H$.

For our next two results, let $I$ be a subgroup of $H$ with $N \leq I \leq H$. Let $V$ be a $k[I]$-module, so that we have the short exact sequence

$$
(0) \rightarrow V J(k[N]) \stackrel{i}{\rightarrow} V \stackrel{\pi}{\rightarrow} V /(V J(k[N])) \rightarrow(0)
$$

in $\operatorname{Mod}(k[I])$ where $i$ denotes the canonic inclusion map and $\pi$ denotes the canonic epimorphism. Since induction is an exact functor [8, VII, Theorem 4.2], we have the short exact sequence

$$
(0) \rightarrow(V J(k[N]))^{H} \stackrel{i^{H}}{\longrightarrow} V^{H} \stackrel{\pi^{H}}{\longrightarrow}(V /(V J(k[N])))^{H} \rightarrow(0)
$$

in $\operatorname{Mod}(k[H])$. For any $g \in G$ and $v \in V$, we have $g J(k[N]) g^{-1}=J(k[N])$ and hence $(v \otimes g) J(k[N])=v J([N]) \otimes g$. Thus we clearly have

LEMMA 2.7. In (2.2), $\operatorname{Im}\left(i^{H}\right)=V^{H} J(k[N])$ and hence $\pi^{H}$ induces a $k[H]$ isomorphism $\lambda: V^{H} /\left(V^{H} J(k[N])\right) \rightarrow(V /(V J(K[N])))^{H}$.

Similarly we have the short exact sequence

$$
(0) \rightarrow \operatorname{Ann}_{V}(J(k[N])) \stackrel{i}{\rightarrow} V \stackrel{\pi}{\rightarrow} V / \operatorname{Ann}_{V}(J(k[N])) \rightarrow(0)
$$

in $\operatorname{Mod}(k[I])$ where $i$ denotes the canonic inclusion map and $\pi$ denotes the canonic epimorphism.

As above, (2.3) yields the short exact sequence

$$
(0) \rightarrow\left(\operatorname{Ann}_{V}(J(k[N]))\right)^{H} \stackrel{i^{H}}{\longrightarrow} V^{H} \stackrel{\pi^{H}}{\longrightarrow}\left(V / \operatorname{Ann}_{V}(J(k[N]))\right)^{H} \rightarrow(0)
$$

in $\operatorname{Mod}(k[H])$. Duality clearly implies

LEMMA 2.8. In (2.4), $\operatorname{Im}\left(i^{H}\right)=\operatorname{Ann}_{V^{H}}(J(k[N]))$.

For our final result of this section, let $M$ be an irreducible $k[N]$-module and let $T=I_{H}(M)=\{g \in H \mid M \otimes g \cong M$ in $\operatorname{Mod}(k[N])\}$, so that $N \leq T \leq H$.

LEMMA 2.9. Let $X$ and $Y$ be finitely generated $k[T]$-modules such that all composition factors $X_{N}$ and $Y_{N}$ are isomorphic to $M$. Then

(a) $\operatorname{Hom}_{k[H]}\left(X^{H}, Y^{H}\right) \cong \operatorname{Hom}_{k[T]}(X, Y)$ over $k$; and

(b) $\operatorname{End}_{k[H]}\left(X^{H}\right) \cong \operatorname{End}_{k[T]}(X)$ as $k$-algebras. 
ProOF. Let $s=|H: T|$ and let $\left\{z_{1}=1, z_{2}, \ldots, z_{s}\right\}$ be a right transversal of $T$ in $H$. Thus $M \otimes z_{i}$ and $M \otimes z_{j}$ are nonisomorphic irreducible $k[N]$-modules for all $1 \leq i, j \leq s$ with $i \neq j$. Clearly $X \cong X \otimes z_{1}=X \otimes 1$ and $Y \cong$ $Y \otimes z_{1}=Y \otimes 1$ in $\operatorname{Mod}(k[T]), M \cong M \otimes z_{1}=M \otimes 1$ in $\operatorname{Mod}(k[N])$ and $\left(Y^{H}\right)_{T}=(Y \otimes 1) \oplus W$ in $\operatorname{Mod}(k[T])$ where $W=\bigoplus_{i=2}^{s}\left(Y \otimes z_{i}\right)$ is a $k[T]$-submodule of $\left(Y^{H}\right)_{T}$. Also $\rho: \operatorname{Hom}_{k[H]}\left(X^{H}, Y^{H}\right) \rightarrow \operatorname{Hom}_{k[T]}\left(X \otimes 1,\left(Y^{H}\right)_{T}\right)$ where $\rho$ denotes restriction to $X \otimes 1$ is a $k$-isomorphism by [8, VII, Theorem 4.5]. Here

$\operatorname{Hom}_{k[T]}\left(X \otimes 1,\left(Y^{H}\right)_{T}\right) \cong \operatorname{Hom}_{k[T]}(X \otimes 1, Y \otimes 1) \oplus \operatorname{Hom}_{k[T]}(X \otimes 1, W)$

and $\operatorname{Hom}_{k[T]}(X \otimes 1, W)$ is a $k$-subspace of $\operatorname{Hom}_{k[N]}\left(X_{N} \otimes 1, W_{N}\right)$. But $W_{N} \cong$ $\bigoplus_{i=2}^{s}\left(Y_{N} \otimes z_{i}\right)$ in $\operatorname{Mod}(k[N])$ and $\operatorname{Hom}_{k[N]}\left(X_{N} \otimes z_{i}, Y_{N} \otimes z_{j}\right)=(0)$ for all $1 \leq$ $i, j \leq s$ with $i \neq j$ since all composition factors of $X \otimes z_{i}$ and $Y \otimes z_{i}$ are isomorphic to $M \otimes z_{i}$ for all $1 \leq i \leq s$. Thus $\operatorname{Hom}_{k[N]}\left(X_{N} \otimes 1, W_{N}\right)=(0)$ and it is now clear that both (a) and (b) hold.

\section{Proofs of the main results.}

ProOF OF LEMMA 1. Assume the notation and hypotheses of Lemma 1. Let $0 \neq x=\sum_{g \in G} x_{g} \in R$ where $x_{g} \in R_{g}$ for all $g \in G$ and assume that $f(x R)=(0)$. Fixing $g \in G$, we have $(0)=f\left(x \beta_{g^{-1}} R_{1}\right)=\lambda\left(T\left(\varphi\left(x_{g} \beta_{g^{-1}} R_{1}\right)\right)\right)$. Thus $\varphi\left(x_{g} \beta_{g^{-1}} R_{1}\right)=(0)$ since $\lambda(T(E))=K$. The hypotheses on $\varphi$ force $x_{g}=0$. Applying [8, VII, Exercise 53], we conclude (a).

Assume the additional hypotheses of (b) and let $x=\sum_{g \in G} r_{g} \beta_{g}$ and $y=$ $\sum_{g \in G} s_{g} \beta_{g}$ be elements of $R$ where $r_{g}, s_{g} \in R_{1}$ for all $g \in G$. Then

$$
\begin{aligned}
f(x y) & =\lambda\left(T\left(\varphi\left(\sum_{g \in G}\left(r_{g} \beta_{g} s_{g^{-1}} \beta_{g^{-1}}\right)\right)\right)\right) \\
& =\sum_{g \in G} \lambda\left(T\left(\varphi\left(r_{g}\left(\beta_{g} s_{g^{-1}} \beta_{g^{-1}}\right)\right)\right)\right)=\sum_{g \in G} \lambda\left(T\left(\varphi\left(\beta_{g} s_{g^{-1}} \beta_{g^{-1}} r_{g}\right)\right)\right)
\end{aligned}
$$

using the fact that $\beta_{g} s_{g^{-1}} \beta_{g^{-1}} \in R_{1}$ for all $g \in G$. Hence

$$
\begin{aligned}
f(x y) & =\sum_{g \in G} \lambda\left(T\left(\varphi\left(\left(s_{g^{-1}} \beta_{g^{-1}} j_{g} \beta_{g}\right)^{\beta_{g}^{-1}}\right)\right)\right) \\
& =\sum_{g \in G} \lambda\left(T\left(\left(\varphi\left(s_{g^{-1}} \beta_{g^{-1}} r_{g} \beta_{g}\right)\right)^{\pi^{*}(g)^{-1}}\right)\right) .
\end{aligned}
$$

However $T=\operatorname{Tr}_{F}^{E}$ and $\operatorname{Tr}_{F}^{E}(e)=\sum_{t \in \pi^{*}(G)} e^{t}$ for all $e \in E$ using the fact that $E / F$ is a finite Galois extension with $\operatorname{Gal}(E / F)=\pi^{*}(G)$ and [9, VIII, Theorem 1.8]. Thus

$$
\begin{aligned}
f(x y) & =\sum_{g \in G} \lambda\left(T\left(\varphi\left(s_{g^{-1}} \beta_{g^{-1}} r_{g} \beta_{g}\right)\right)\right) \\
& =\lambda\left(T\left(\varphi\left(\sum_{g \in G}\left(s_{g^{-1}} \beta_{g^{-1}} r_{g} \beta_{g}\right)\right)\right)\right)=f(y x) .
\end{aligned}
$$

Now [8, VII, Exercise 54] completes the proof of (b).

A PROOF OF PROPOSITION 2. Assume the hypotheses of Proposition 2. Let $R_{1}=\bigoplus_{i=1}^{n} B_{i}$ be the decomposition of $R_{1}$ into ideals such that each $B_{i}$ is a simple $K$-algebra for all $1 \leq i \leq n$. Let $1=\sum_{i=1}^{n} e_{i}$ where $e_{i} \in B_{i}=e_{i} R_{1}$ for all $1 \leq i \leq n$. 
Then $\mathscr{I}=\left\{e_{i} \mid 1 \leq i \leq n\right\}$ is the set of primitive central idempotents of $R_{1}$ and $\operatorname{Gr} U(R)$, acting by conjugation, permutes $\mathscr{I}$. Clearly $U\left(R_{1}\right)=\bigoplus_{i=1}^{n} U\left(B_{i}\right)$ is contained in the kernel of this action. Thus we may view $G$ as permuting $\mathscr{I}$. Next let $\mathscr{I}=\bigcup_{j=1}^{r} \mathscr{I}_{j}$ be the $G$-orbit decomposition of $\mathscr{I}$. Set $f_{j}=\sum_{e_{i} \in \mathcal{F}_{j}} e_{i}$ for all $1 \leq j \leq r$. Then $\left\{f_{j} \mid 1 \leq k \leq r\right\}$ is a set of $G$-fixed orthogonal idempotents of $Z\left(R_{1}\right)$ such that $1=\sum_{j=1}^{r} f_{j}$. Since a finite direct sum of symmetric $K$-algebras is a symmetric $K$-algebra, Lemma 2.4 implies that it suffices to assume that $G$ acts transitively on $\mathcal{I}$.

Set $e=e_{1}, f=1-e, B=B_{1}=e R_{1}, E=Z(B)$ and $H=\operatorname{Stab}_{G}(e)$. Clearly $E$ is a field, $K \cong K e=e K \subseteq E, R_{H}=\bigoplus_{h \in H} R_{h}$ is an $H$-crossed product $K$-algebra, $\{e, f\}$ is a set of $H$-fixed orthogonal central idempotents of $R_{1}$ such that $1=e+f, \operatorname{Gr} U\left(R_{H}\right)=\bigcup_{h \in H}\left(U(R) \cap R_{h}\right)$ acts by conjugation on $B$ and $E$ and $U\left(R_{1}\right)=\bigoplus_{i=1}^{n} U\left(B_{i}\right)$ acts trivially by conjugation on $E$. Thus conjugation induces a group homomorphism $\Pi: H \rightarrow \operatorname{Aut}(E)$ and Lemma 2.4 implies that $R_{H}=\left(e R_{H}\right) \oplus\left(f R_{H}\right)$ where $e R_{H}$ and $f R_{H}$ are $H$-crossed product $K$-algebras, etc. Here $\left(e R_{H}\right)_{1}=e R_{1}=B$ is a simple $K$-algebra with $Z(B)=E$. Clearly $\Pi: H \rightarrow$ $\operatorname{Aut}(E)$ is precisely the homomorphism induced by conjugation of $\operatorname{Gr} U\left(e R_{H}\right)$ on $E$. Let $F=E^{H}$ denote the $H$-fixed subfield of $E$, so that $K \cong K e=e K \subseteq F$. Also let $T=\operatorname{Tr}_{F}^{E}: E \rightarrow F$ and $0 \neq \lambda \in \operatorname{Hom}_{K}(F, K)$ be as in Lemma 1. Viewing $B$ as a finite dimensional (simple) $E$-algebra where $E=Z(B)$, the reduced trace $\operatorname{tr}_{B / E} \in \operatorname{Hom}_{E}(B, E)$ is defined (cf. [2, §7D]). Moreover $\operatorname{tr}_{B / E}(x y)=\operatorname{tr}_{B / E}(y x)$ for all $x, y \in B$ and $\operatorname{Ker}\left(\operatorname{Tr}_{B / E}\right)$ contains no nonzero right ideal of $B$ by [2, Corollary 7.6 and Proposition 7.41]. Also $\operatorname{tr}_{B / E}\left(x^{u}\right)=\operatorname{tr}_{B / E}(x)^{u}$ for all $x \in B$ and all $u \in \operatorname{Gr} U\left(e R_{H}\right)$ by Lemma 2.3. Define $f: e R_{H} \rightarrow K$ as in Lemma 1 , so that $f \in \operatorname{Hom}_{K}\left(e R_{H}, K\right),\left.f\right|_{B}=\lambda \circ \operatorname{Tr}_{F}^{E} \circ \operatorname{tr}_{B / E} \in \operatorname{Hom}_{K}(B, K), \operatorname{Ker}\left(\left.f\right|_{B}\right)$ contains no nonzero right ideal of $B$ and

$$
f\left(x^{u}\right)=f(x) \text { for all } x \in B \text { and all } u \in \operatorname{Gr} U\left(e R_{H}\right) .
$$

Let $\left\{x_{1}=1, x_{2}, \ldots, x_{n}\right\}$ be a choice of right coset representatives of $H$ in $G$, so that $G=\bigcup H x_{i}$. Also set $\beta_{i}=\beta_{x_{i}}$ for all $1 \leq i \leq n$ and choose the notation so that $B_{1}^{\beta_{i}}=B_{i}$ for all $1 \leq i \leq n$. Clearly $\alpha_{i}: B_{1} \rightarrow B_{i}$ defined by: $\alpha_{i}(b)=\beta_{i}^{-1} b \beta_{i}$ for all $b \in B=B_{1}$ is a $K$-algebra isomorphism for all $1 \leq i \leq n$.

Define $\varphi: R_{1}=\bigoplus_{i=1}^{n} B_{i} \rightarrow K$ by: if $y=\sum_{i=1}^{n} y_{i} \in R_{1}$ for unique elements $y_{i} \in B_{i}$ for all $1 \leq i \leq n$, set $\varphi(y)=\sum_{i=1}^{n} f\left(\beta_{i} y_{i} \beta_{i}^{-1}\right)$. Clearly $\varphi \in \operatorname{Hom}_{K}\left(R_{1}, K\right)$, $\varphi(x y)=\varphi(y x)$ for all $x, y \in R_{1}$ and $\operatorname{Ker}(\varphi)$ contains no nonzero right ideal of $R_{1}$. Fix $u \in \operatorname{Gr} U(R), 1 \leq j \leq n$ and $z \in B_{j}$. Clearly there is a unique $1 \leq k \leq n$ such that $B_{j}^{u}=u^{-1} \beta_{j}^{-1} B_{1} \beta_{j} u=B_{k}$ and hence $\beta_{j} u=\gamma \beta_{k}$ for a unique $\gamma \in U(R) \cap R_{h}$ and for a unique $h \in H$. Thus

$$
\varphi\left(z^{u}\right)=\varphi\left(u^{-1} z u\right)=f\left(\beta_{k} u^{-1} z u \beta_{k}^{-1}\right)=f\left(\gamma^{-1} \beta_{j} z \beta_{j}^{-1} \gamma\right) .
$$

But $\gamma=e \gamma+f \gamma, \gamma^{-1}=e \gamma^{-1}+f \gamma^{-1}$ and $B=B_{1}=e R_{1}$, so that $(*)$ implies $\varphi\left(z^{u}\right)=f\left(\left(\beta_{j} z \beta_{j}^{-1}\right)^{e \gamma}\right)=f\left(\beta_{j} z \beta_{j}^{-1}\right)=\varphi(z)$. It follows that $\varphi\left(x^{v}\right)=\varphi(x)$ for all $x \in R_{1}$ and all $v \in \operatorname{Gr} U(R)$. Now Lemma 1(b) with $E=K 1$ yields the desired conclusion.

For the remainder of the paper, we shall assume the notation of the final segment of $\S 1$ and we set $G=H / N$. 
A PROOF OF THEOREM 4. As is well known, $k[H]$ can be viewed as a $G=$ $H / N$-crossed product $k$-algebra where $(k[H])_{N h}=\bigoplus_{x \in N h} k x$ for all $h \in H$. Then $k[H]_{N}=k[N], J(k[N]) k[H]$ is a $G$-graded ideal with $(J(k[N]) k[H])_{N}=J(k[N])$ (cf. [7, Lemmas 2.4-2.7]) and $k[H] /(J(k[N]) k[H])$ is a $G$-graded $k$-algebra with

$$
(k[H] /(J(k[N]) k[H]))_{N h}=\left(k[H]_{N h}+J(k[N]) k[H]\right) /(J(k[N]) k[H])
$$

for all $h \in H$. Since

$$
\begin{aligned}
& \left(k[H]_{N}+J(k[N]) k[H]\right) /(J(k[N]) k[H]) \\
& \quad=(k[N]+J(k[N]) k[H]) /(J(k[N]) k[H]) \cong k[N] / J(k[N])
\end{aligned}
$$

as $k$-algebras and since $k[N] / J(k[N])$ is a semisimple $k$-algebra, Proposition 2 yields the desired conclusion.

A PROOF OF THEOREM 5. Assume the hypotheses of Theorem 5 and observe that if $X$ is any $k[N]$-module and $h \in H$, then $(X \otimes h)^{H} \cong X^{H}$ in $\operatorname{Mod}(k[H])$. It follows that we may assume that there are a positive integer $s$, irreducible $k[N]$ modules $M_{1}, \ldots, M_{s}$ and positive integers $r_{1}, \ldots, r_{s}$ such that $W=\bigoplus_{i=1}^{s}\left(r_{i} M_{i}\right)$ and such that for any $1 \leq i, j \leq s$ and any $x, y \in H, M_{i} \otimes x \cong M_{j} \otimes y$ in $\operatorname{Mod}(k[N])$ implies that $i=j$.

Let $\mathscr{T}$ be a transversal of $N$ in $H$ with $\mathscr{T} \cap N=\{1\}$. Suppose that $1 \leq i, j \leq s$ with $i \neq j$. Then $\operatorname{Hom}_{k[H]}\left(\left(r_{i} M_{i}\right)^{H},\left(r_{j} M_{j}\right)^{H}\right) \cong \operatorname{Hom}_{k[N]}\left(r_{i} M_{i},\left(r_{j} M_{j}^{H}\right)_{N}\right)$ by $[\mathbf{8}$, VII, Theorem 4.5]. Thus

$$
\operatorname{Hom}_{k[H]}\left(\left(r_{i} M_{i}\right)^{H},\left(r_{j} M_{j}\right)^{H}\right) \cong r_{i} r_{j}\left(\bigoplus_{x \in \mathscr{T}} \operatorname{Hom}_{k[N]}\left(M_{i}, M_{j} \otimes x\right)\right)=(0)
$$

since $M_{i}$ and $M_{j} \otimes x$ are irreducible and nonisomorphic $k[N]$-modules for all $x \in H$. As is well known, this fact implies that

$$
\operatorname{End}_{k[H]}\left(W^{H}\right) \cong \bigoplus_{i=1}^{s} \operatorname{End}_{k[H]}\left(\left(r_{i} M_{i}\right)^{H}\right)
$$

as $k$-algebras. Since a direct sum of symmetric $k$-algebras is also a symmetric $k$ algebra by [8, VII, Exercise 54], it suffices to assume that $s=1$. Set $r=r_{1}$, and $M=M_{1}$, so that $W=r M$, and let $I=\{h \in H \mid M \otimes h \cong M$ in $\operatorname{Mod}(k[N])\}$, so that $N \leq I \leq H$.

Here $W^{H}=(r M)^{H} \cong r\left(M^{H}\right)$ and hence $\operatorname{End}_{k[H]}\left(V^{H}\right) \cong\left(\operatorname{End}_{k[H]}\left(M^{H}\right)\right)_{r}$ as $k$-algebras, where $\left(\operatorname{End}_{k[H]}\left(M^{H}\right)\right)_{r}$ denotes the full $r \times r$ matrix $k$-algebra over $\operatorname{End}_{k[H]}\left(M^{H}\right)$. Applying [8, VII, Exercise 48], it suffices to assume that $r=1$ and $W=M$ is irreducible in $\operatorname{Mod}(k[N])$. As is well known, $\operatorname{End}_{k[H]}\left(M^{H}\right) \cong$ $\operatorname{End}_{k[I]}\left(M^{I}\right)$ as $k$-algebras (cf. Lemma 2.9(b)). Thus it suffices to assume that $H=I$. Then $\operatorname{End}_{k[H]}\left(M^{H}\right)$ can be viewed as a $G=H / N$-crossed product $K$ algebra with $\left(\operatorname{End}_{k[H]}\left(M^{H}\right)\right)_{1} \cong \operatorname{End}_{k[N]}(M)$ by $[4, \S \S 4-5]$. Since $\operatorname{End}_{k[N]}(M)$ is a division $k$-algebra by Schur's Lemma, an application of Proposition 2 completes our proof of Theorem 5 .

A PROOF OF PROPOSITION 6. Let $W$ and $r$ be as in Proposition 6 and let $0 \leq j \leq r-1$. Clearly $W \mid\left(W_{N}\right)^{H}$ in $\operatorname{Mod}(k[H])$ by [6, II, Theorem 3.8]. Thus

$$
\left(\left(W J(k[N])^{j}\right) /\left(W J(k[N])^{j+1}\right) \mid\left(\left(\left(W_{N}\right)^{H}\right) J(k[N])^{j}\right) /\left(\left(\left(W_{N}\right)^{H}\right) J(k[N])^{j+1}\right)\right.
$$


in $\operatorname{Mod}(k[H])$ by Lemma 2.1. Also $\left(\left(\left(W_{N}\right)^{H}\right) J(k[N])^{j}\right) /\left(\left(\left(W_{N}\right)^{H}\right) J(k[N])^{j+1}\right)$ is a $G$-graded module for the $G$-crossed product $k$-algebra $k[H] /(J(k[N]) k([H])$ by [7, Lemmas 2.4, 2.6-2.7 and Remark 2.5]. Since $(k[H] /(J(k[N]) k[H]))_{1} \cong$ $k[N] / J(k[N])$ as $k$-algebras, Lemma 2.5 and Theorem 4 imply the desired conclusions.

A PROOF OF THEOREM 7. Assume the hypotheses of Theorem 7. Clearly we have a short exact sequence

$$
(0) \rightarrow P(M) J(k[N]) \stackrel{i}{\rightarrow} P(M) \stackrel{\pi}{\rightarrow} M \rightarrow(0)
$$

where $i$ denotes the canonic inclusion map and $\pi$ denotes an epimorphism in $\operatorname{Mod}(k[N])$. Since induction is an exact functor [8, VII, Theorem 4.2], we obtain the following short exact sequence

$$
(0) \rightarrow((P(M) J(k[N])))^{H} \stackrel{i^{H}}{\longrightarrow} P(M)^{H} \stackrel{\pi^{H}}{\longrightarrow} M^{H} \rightarrow(0)
$$

in $\operatorname{Mod}(k[H])$. Lemma 2.7 implies that $\operatorname{Im}\left(i^{H}\right)=P(M)^{H} J(k[N])$ and $\pi^{H}$ induces a $k[H]$-isomorphism $\lambda:\left(P(M)^{H}\right) /\left(P(M)^{H} J(k[N])\right) \rightarrow M^{H}$. It follows that $\mathscr{H}\left(P(M)^{H}\right) \cong \mathscr{H}\left(M^{H}\right)$ in $\operatorname{Mod}(k[H])$. Since $P(M)^{H}$ is a projective $k[H]$-module, [8, VII, Theorem 10.9(a)] yields (a). Applying Lemma 2.1(b), the isomorphism $\lambda$ yields (b).

Here $\mathscr{S}(P(M))=\operatorname{Ann}_{P(M)}(J(k[N])) \cong M$ in $\operatorname{Mod}(k[N])$ and hence (a) and Lemmas 2.8 and 2.1(c) imply:

$$
M^{H} \cong \bigoplus_{L \in \operatorname{Irr}(k[H] \mid M)}\left(\operatorname{mult}\left(L \text { in } \mathscr{H}\left(M^{H}\right)\right) \operatorname{Ann}_{P(L)}(J(k[N]))\right) .
$$

Fix $L \in \operatorname{Irr}(k[H] \mid M)$. We noted above that

$$
\mathscr{H}(P(L) /(P(L) J(k[N]))) \cong L \cong \mathscr{S}\left(\operatorname{Ann}_{P(L)}(J(k[N]))\right) .
$$

Thus $P(L) /(P(L) J(k[N]))$ and $\operatorname{Ann}_{P(L)}(J(k[N]))$ are indecomposable $k[H]$ modules. Using (b), (3.1) and the Krull-Schmidt Theorem, (c) holds once we prove

$$
\mathscr{S}(P(L) /(P(L) J(k[N]))) \cong L \quad \text { in } \operatorname{Mod}(k[H]) .
$$

Since $P(L) \mid P(M)^{H}$, Proposition 6 implies that $P(L) /(P(L) J(k[N]))$ is a projective indecomposable module over the symmetric $k$-algebra $k[H] /(J(k[N]) k[H])$. Since $\mathscr{H}(P(L) /(P(L) J(k[N]))) \cong L[8$, VII, Theorem 11.6(c)] yields (3.2) and we are done.

A PROOF OF COROLlary 8. Let $L \in \operatorname{Irr}(k[H])$. Assume (a), so that $L \mid\left(L_{N}\right)^{H}$. Hence there is an irreducible $k[N]$-module $M$ such that $M \mid L_{N}$ and $L \mid M^{H}$. Then Theorem 7 implies (b). Assume (b) and choose an irreducible $k[N]$-module $M$ such that $M \mid L_{N}$. Then Theorem 7 implies that $L \mid M^{H}$ and (a) holds.

A PROOF OF COROLlaRY 9. Clearly (a) implies (b) by Theorem 7. Assume (b). Then $\left(1_{N}\right)^{H}$ is a completely reducible $k[H]$-module. Thus $k[H / N]$ is a semisimple $k$-algebra and Maschke's Theorem yields (c). Next assume (c) and let $L \in \operatorname{Irr}(k[H])$. Then $L$ is $k[N]$-projective by [8, VII, Theorem 7.7(b)] and hence $P(L) J(k[N])=P(L) J(k[H])$ by Corollary 8 . Since

$$
k[H]_{k[H]} \cong \bigoplus_{L \in \operatorname{Irr}(k[H])}\left(\text { mult } L \text { in } \mathscr{H}\left(1^{H}\right)\right) P(L)
$$


by Theorem 7(a), for example, (a) is immediate. The last statement follows from Frobenius reciprocity [8, VII, Theorem 4.5], Theorem 7 and (a).

A PROOF OF COROLlaRY 10. With the hypotheses of Corollary 10, apply Theorem 7 with $M=1_{N}$ to conclude that $P(L) /(P(L) J(k[N]))$ is isomorphic to an indecomposable component of $\left(1_{N}\right)^{H}$ and all indecomposable components $X$ of $\left(1_{N}\right)^{H}$ with $\mathscr{H}(X) \cong L$ in $\operatorname{Mod}(k[H])$ satisfy $X \cong P(L) /(P(L) J(k[N]))$ in $\operatorname{Mod}(k[H])$. Since $Q(L) \mid\left(1_{N}\right)^{H}$ and $\mathscr{H}(Q(L)) \cong L$ in $\operatorname{Mod}(k[H])$, we conclude (a). Clearly $A(N)=\operatorname{Ann}_{k[N]}\left(1_{N}\right) \geq J(k[N])$ and $N$ acts trivially on $Q(L)$, so that $P(L) A(N) \leq P(L) J(k[N])$. Thus (b) holds and our proof is complete.

\section{REFERENCES}

1. H. Cartan and S. Eilenberg, Homological algebra, Princeton Univ. Press, Princeton, N.J., 1956.

2. C. W. Curtis and I. Reiner, Methods of representation theory, Vol I, Wiley, New York, 1981.

3. E. C. Dade, Isomorphisms of Clifford extensions, Ann. of Math. 92 (1970), 375-433.

4. —_, Group-graded rings and modules, Math. Z. 174 (1980), 241-262.

5. _ The equivalence of various generalizations of group rings and modules, Math. Z. 181 (1982), 335-344.

6. W. Feit, The representation theory of finite groups, North-Holland, New York, 1982.

7. M. .E. Harris, Filtrations, stable Clifford theory and group-graded rings and modules, Math. Z. 196 (1987), 497-510.

8. B. Huppert and N. Blackburn, Finite groups II, Springer-Verlag, Berlin, 1982.

9. S. Lang, Algebra, 2nd ed., Addison-Wesley, Reading, Mass., 1984.

10. B. Pareigis, Einige Bemerkungen über Frobenius-Erweiterungen, Math. Ann. 153 (1964), 1-13. 55455

School of Mathematics, University of Minnesota, Minneapolis, Minnesota 